Vietnam Journal of Mechanics, VAST, Vol. 40, No. 1 (2018), pp. 1 -13

DOI:10.15625/0866-7136/8488

\title{
SUPER CAVITY MODEL WITH THE COUPLING REACTION OF SLENDER BODY MOTION AND WATER FLOW
}

\author{
Tran Thu Ha ${ }^{1,2,3, *}$, Nguyen Duc Thuyen ${ }^{4}$, Nguyen Thai Dung ${ }^{4}$, Duong Ngoc Hai ${ }^{2,3}$ \\ ${ }^{1}$ Institute of Mechanics, VAST, Hanoi, Vietnam \\ ${ }^{2}$ Graduate University of Science and Technology, VAST, Hanoi, Vietnam \\ ${ }^{3}$ VNU University of Engineering and Technology, Hanoi, Vietnam \\ ${ }^{4}$ Le Quy Don University, Hanoi, Vietnam \\ *E-mail: ttha@imech.vast.vn \\ Received July 13, 2016
}

\begin{abstract}
On the imperfect water entry, a high-speed slender body moving in the forward direction, rotates inside the cavity. The body's motion makes super cavity phenomena in the water flow. The water velocity and pressure fields interact during the body's motion. In this paper, the coupling simulation model is a combination of two sub-models: In the first sub-model, the motion of slender body running very fast underwater is simulated. The equation system of this sub-model is solved by Runge-Kutta method; In the second sub-model, the water flow and pressure field under reaction of very fast slender body motion are simulated by CFD model. The simulation results of this coupled model are compared with experiments based on magnitudes of velocity $U$ by $x_{0}$ direction and error percents for cavity diameter and length.
\end{abstract}

Keywords: Cavity model, Runge-Kutta method, CFD model, coupling model.

\section{INTRODUCTION}

The cavity phenomena occur when a slender body runs very fast under water (velocity is higher than $50 \mathrm{~m} / \mathrm{s}$ ). In hydrodynamic applications cavitation is the appearance of vapor bubbles and pockets inside homogeneous liquid medium. This phenomenon occurs because the pressure is reduced to the vapor pressure limit. Cavitation is an important phenomenon which can have a profound effect on the performance of a number of devices. Many of Computational Fluid Dynamics (CFD) models developed for cavitation involve the use of an equation of state to express the mixture density as a function of local pressure. This modeling approaches are attractive because they can be integrated into a basic-functionality CFD code without much effort. However, it must also be recognized that these approaches are overly simplistic in their assumption of equilibrium

(C) 2018 Vietnam Academy of Science and Technology 
thermodynamics. By this, we mean that as the flow condition changes, the two-phase fluid is assumed to instantaneously reach its equilibrium thermodynamic state.

We will study super cavity appearing by the very fast movement of slender body underwater. When the body head called by cavitator is directly touching with water, the gas layer may cover partial or full body depending on the design of body form. The body rotates about its nose. The form of body's nose can be differently chosen such as: sharp, hemisphere, plate disk... For simple calculation we choose cavitator formed by the plate disk with diameter $d_{c}$ (see Fig. 1). The body is consisted of two parts: the cone top and cylinder part with the diameter $d$. In this paper the mathematical model of slender body running very fast underwater is the combination of two equation sub-systems:

1) The first equation sub-system describes rigid body's motion in cavity. This equation sub-system is solved by Runge-Kutta method. The obtaining result set contains the body velocities. In the super cavity model the following assumptions are (see [1]):

- The motion of the slender body is confined to a plane;

- The slender body rotates about its nose;

- The effect of gravity on the dynamics of this body is negligible;

- The motion of the slender body is not influenced by the presence of gas, water vapor or water drops in the cavity;

2) The second equation sub-system describes flow's state of mixture fluid-gas with the cavitation phenomena. Recently, the viscous flow models, which regard the cavitating flow as the bubble layer containing spherical bubbles, were introduced to provide highly accurate calculations. In the viscous flow models, the Navier-Stokes equation including cavitation bubble is solved in conjunction with Rayleigh-Plesset's equation governing the change in the bubble radius. The $k-\varepsilon$ equations are solved to describe the turbulence term in Navier-Stokes equation. This equation system is solved by CFD model. The getting result set is the pressure fluctuation, velocity fields of fluid and mixture fluid-gas.

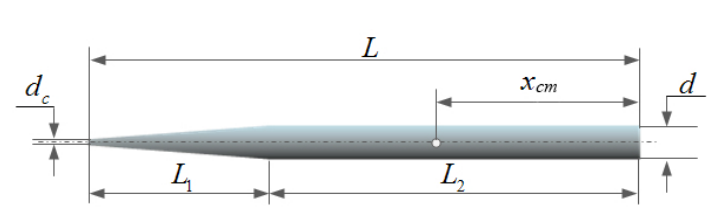

Fig. 1. Slender body geometry
- $L$ is the length of the slender body;

- $L_{2}$ is the body's length of cylinder part;

- $L_{1}$ is the body's length of cone top part;

$-d$ is the body's diameter;

$-d_{c}$ is the body's nose diameter;

- $x_{c m}$ is the dimension between the center of mass and bottom of body.

For the first of sub-model in [1] the cavity number $\sigma$ is not influenced by the exciting flow pressure field arising by body's movement effect to fluid. For the second sub-model in the body is not moving and fluid flow is flowing with constant velocity.

In this paper two of these equation sub-systems are connected by exchange of velocities at the nose's rigid body and cavity number $\sigma$, that is influenced by the exciting ambient flow pressure. Available experimental measurements are used for comparison with simulation results. 


\section{THEORETICAL FORMULATION}

The mathematical model is the combination of the following two sub-models:

- Mathematical sub-model is simulating body's motion: Using the number cavity the body's velocities are solved by this model.

- The sub-model simulating flow velocity and pressure field with cavitation: Applying the body's velocities at the body's nose the water flow velocity and pressure field are solved by this model. Then, the cavity number $\sigma$ (see formula (4)) is obtained.

\subsection{Mathematical sub-model simulated motion of slender body running very fast un- der water}

To describe the body's motion, a body-fixed coordinate system shown in Fig. 2 is chosen. $\left(x_{0}, y_{0}, z_{0}\right)$ is the inertial reference frame with origin at $O$ and $(x, y, z)$ is the noninertial reference frame with origin at $\mathrm{A}$, that is the tip of the slender body.
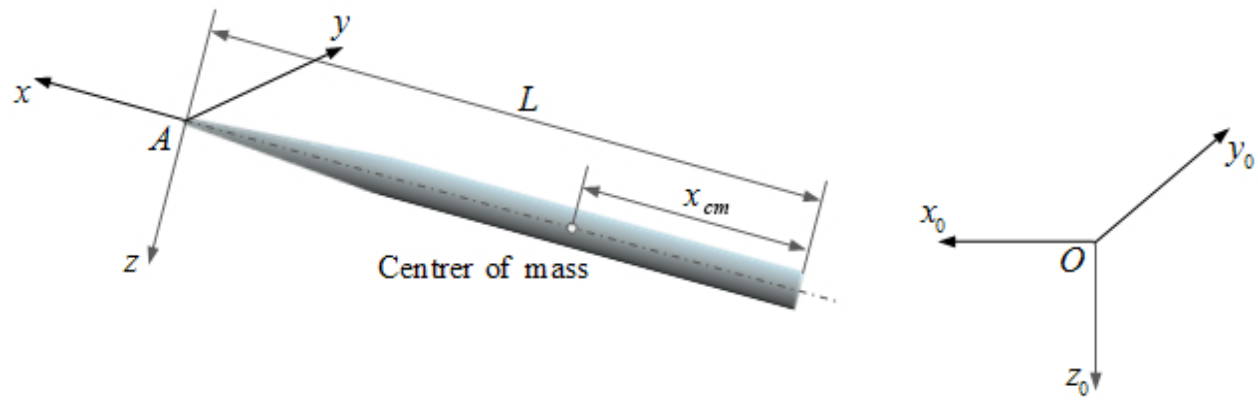

Fig. 2. Axes of body and inertial frames

The $x$-axis coincides with the longitudinal axis of the slender body. The components of velocity at the point $A$ along $x$ and $z$ direction are $U_{F}$ and $W_{F}$, respectively. The components of velocity at the point $A$ along $x_{0}$ and $z_{0}$ direction are $U$ and $W$, respectively. The angular velocity about $y_{0}$ axis is $Q$. The orientation angle of the body with respect to the $y_{0}$ axis is $\vartheta$.

The relationship between body and fixed inertial velocities is described by the following formula (see [2])

$$
\left\{\begin{array}{l}
U_{F}=U \cos \vartheta+W \sin \vartheta \\
W_{F}=-U \sin \vartheta+W \cos \vartheta \\
\dot{\vartheta}=Q \\
\vartheta(0)=\vartheta_{0}
\end{array}\right.
$$

The mathematic cavity model (see [1]) is used to describe the motion of slender body under water in cavity. The motion of slender body in both phases is written by the following equations:

- Phase 1: For $U^{2} \gg W^{2}$ and $\rho_{l} A_{c} k(U, W, h) U^{2} \gg 2 m L Q^{2}$ 
The equations can be written as

$$
\left\{\begin{array}{l}
\frac{\partial U}{\partial t}=-\frac{1}{2 m} \rho_{l} k(U, W, h) A_{c} U^{2} \\
\frac{\partial W}{\partial t}=Q U \\
\frac{\partial Q}{\partial t}=0 \\
\frac{\partial h}{\partial t}=W \\
\frac{\partial \vartheta}{\partial t}=Q \\
U(0)=U_{0} ; W(0)=W_{0} ; Q(0)=Q_{0} ; h(0)=h_{0} ; \vartheta(0)=\vartheta_{0}
\end{array}\right.
$$

- Phase 2: For $U^{2} \gg W^{2}$ and $\rho_{l} A_{c} k(U, W, h) U^{2} \gg 2 m L Q^{2}$

The equations can be written as

$$
\left\{\begin{array}{l}
\frac{\partial U}{\partial t}=-\frac{1}{2 m} \rho_{l} k(U, W, h) f\left(A_{c}, r, l_{k}, \theta\right) U^{2} \\
\frac{\partial W}{\partial t}=K W^{2}\left[\left(M_{1} l_{k}\right)+M_{2}\left(l_{k} x_{c m}\right)\left(L-x_{c m}\right)\right]+2 W\left[Q M_{2}\left(L l_{k} x_{c m}\right)\left(L-x_{c m}\right)\right]+Q U \\
\frac{\partial Q}{\partial t}=-K M_{2}\left[W^{2}\left(l_{k} x_{c m}\right)+2 Q W\left(L l_{k} x_{c m}\right)\right] \\
\frac{\partial h}{\partial t}=W \\
\frac{\partial \vartheta}{\partial t}=Q
\end{array}\right.
$$

where: $\theta$ is the angle of slender body during impact with the cavity wall: $\tan \theta \approx \frac{W}{U}$ or $\theta \approx \arctan \frac{W}{U} ; M_{1}=-\frac{K \rho_{l} d}{m} ; M_{2}=\frac{K \rho_{l} d}{I} ;$

$f\left(A_{c}, r, l_{k}, \theta\right)=A_{C}+r^{2} \cos ^{-1}\left(\frac{r-l_{k} \tan \theta}{r}\right)-\left(r-l_{k} \tan \theta\right) \sqrt{d l_{k} \tan \theta} ;$

$k(U, W, h)=k_{1} C_{D 0}(1+\sigma) \cos ^{2} \alpha($ see $[3,4])$;

$C_{D 0}=0.82$

$\alpha$ is the angle between flow direction and body's direction in moving: $\cos \alpha \approx \frac{U}{\sqrt{U^{2}+W^{2}}}$

$l_{k}$ is the wetted length of the body;

$k_{1}, K$ are parameters;

$h$ is the water depth between the body's position and water free surface;

$\rho_{l}$ is the mass density of water;

$m$ is the mass of the slender body;

$\sigma$ is the cavity number:

$$
\sigma=\frac{p-p_{v}}{0.5 \rho_{l} U_{F}^{2}} \quad \text { (see [5]) }
$$


$p$ is the ambient flow pressure;

$I$ is the moment of inertia of the body about an axis parallel to the $y$ axis and passing through its center of mass;

$r=\frac{d}{2}$ is the body's radius;

$A_{c}=\frac{\pi d_{c}^{2}}{4}$ is the area of the cavitator;

$r_{c}=\frac{d_{c}}{2}$ is the cavitator radius;

$g=9.81 \mathrm{~m} / \mathrm{s}$ is the gravity acceleration;

$p_{v}$ is the vapor pressure of water.

To get the above equations (2)-(3) the following condition is needed: $\frac{l_{k}}{L} \ll 1$.

The cavity shape is assumed to be an ellipse (actually, it is an ellipsoid in 3D) (see [1, $4,6]$ ). The shape and size of the elliptic cavity are characterized by its maximum diameter and its length (see Fig. 3)

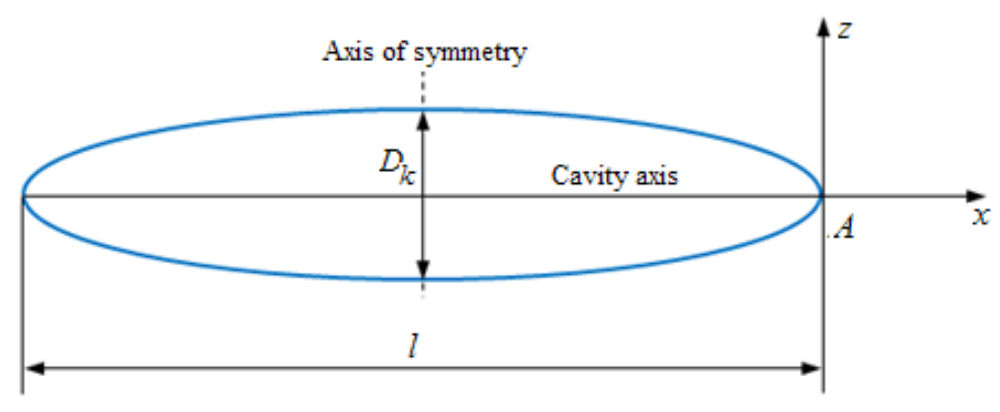

Fig. 3. Shape of the cavity

The geometry of the cavity is given by the following formula (see $[1,4,6]$ )

$$
\frac{\left(x+\frac{l}{2}\right)^{2}}{\left(\frac{l}{2}\right)^{2}}+\frac{z^{2}}{\left(\frac{D_{k}}{2}\right)^{2}}=1,
$$

where the maximum diameter $D_{k}$ and length $l$ of the cavity shape are given by the following formulas

$$
\begin{gathered}
D_{k}=d_{c} \sqrt{\frac{C_{D 0}(1+\sigma)}{\sigma}} ; \\
l=\frac{d_{c}}{\sigma} \sqrt{\ln \left(\frac{1}{\sigma}\right)} .
\end{gathered}
$$


Eqs. (2)-(3) can be rewritten as follows

$$
\left\{\begin{array}{l}
\frac{\partial X}{\partial t}=A(X) \\
X(0)=X_{0}
\end{array}\right.
$$

where

$$
\begin{aligned}
& X=(U, W, Q, h, \vartheta)^{T} \text { is unknown state function vector and } X_{0}=\left(U_{0}, W_{0}, Q_{0}, h_{0}, \vartheta_{0}\right)^{T} ; \\
& A(X)=\left[A_{1}(X), A_{2}(X), A_{3}(X), W, Q\right]^{T} \text { with: } \\
& A_{1}(X)= \begin{cases}-\frac{1}{2 m} \rho_{l} k(U, W, h) A_{c} U^{2} & \text { in the first phase } \\
-\frac{1}{2 m} \rho_{l} k(U, W, h) f\left(A_{c}, r, l_{k}, \theta\right) U^{2} & \text { in the second phase }\end{cases} \\
& A_{2}(X)= \begin{cases}Q U & \text { in the first phase } \\
K C_{1} W^{2}+K C_{2} W+Q U & \text { in the second phase } \\
Q U & \text { in the first phase } \\
A_{3} W^{2}+C_{4} Q & \text { in the second phase }\end{cases}
\end{aligned}
$$

with th

$C_{1}=M_{1} l_{k}+M_{2} l_{k} x_{c m}\left(L-x_{c m}\right) ;$

$C_{2}=2 M_{2} L x_{c m} l_{k}\left(L-x_{c m}\right)$;

$C_{3}=-M_{2} l_{k} x_{c m}$

$C_{4}=-M_{2} L l_{k} x_{c m}$.

To solve Eq. (5) the Runge-Kutta method is used.

\subsection{Mathematical sub-model simulated water flow under reaction of very fast slender body motion}

Cavitating flow is very sensitive to the formation and transport of vapor bubbles, the turbulent fluctuations of pressure and velocity and to the magnitude of non-condensable gases, which are dissolved or ingested in the operating liquid [7,8]. Although basic cavitation theoretical studies deal with bubble (or bubble clouds) dynamics by solving the vapor-liquid interface, most of practical cavitating flows are approached using a homogeneous flow theory. The main idea is to consider a single variable density fluid, without explicit phase interfaces. This model has emerged after examining available experimental investigations, as well as by numerical studies over the past decade can be found in [9], where various Reynolds Averaged Navier-Stokes (RANS) solvers have been modified to account for the second phase (vapor and gas) dynamics.

The mixture model is used in the current work for the numerical simulation of cavitating flows with the CFD model. In this model, the flow is assumed to be thermal and dynamic equilibrium at the interface where the flow velocity is assumed to be continuous.

The mixture is a hypothetical fluid with variable density written as follows: $\rho_{m}=$ $\alpha \rho_{v}+(1-\alpha) \rho_{l}$. This density is ranging from fluid density $\rho_{l}$ for $\alpha=0$ to vapor density $\rho_{v}$ for $\alpha=1$.

As already indicated the cavitating flow is modeled as a mixture of two species, that is vapor and liquid behaving as a single one, where both phases share the same velocity as well as pressure fields. In the case of the RANS approach to turbulence, employing 
the eddy viscosity models and assuming both liquid and vapor phases incompressible, turbulent cavitating flows can be described by the following set of governing equations:

The continuity equation of mixture fluid is written in the form (see [10])

$$
\nabla U_{m}=\dot{m}\left(\frac{1}{\rho_{v}}-\frac{1}{\rho_{l}}\right)
$$

with $U_{m}=\left(u_{1}, u_{2}, u_{3}\right)$.

The momentum equation takes the form (see [5])

$$
\frac{\partial\left(\rho_{m} u_{i}\right)}{\partial t}+\frac{\partial\left(\rho_{m} u_{j}\right)}{\partial x_{j}}=\rho_{m} F_{i}-\frac{\partial P}{\partial x_{i}}+\frac{\partial \tau_{i j}}{\partial x_{j}}
$$

with

$$
\begin{aligned}
& \tau_{i j}=\left(\mu_{m}+\mu_{m t}\right)\left(\frac{\partial u_{i}}{\partial x_{j}}+\frac{\partial u_{j}}{\partial x_{i}}\right), \\
& \mu_{m}=(1-\alpha) \mu_{l}+\alpha \mu_{v}, \\
& \left.\mu_{m t}=\frac{\rho_{m} C_{\mu} k^{2}}{\varepsilon} \text { (see }[11,12]\right) .
\end{aligned}
$$

To find the turbulence coefficient $\mu_{m t}$ a two-equation turbulence model with standard wall functions has been implemented to provide closure. As with velocity, the turbulence scalars are interpreted as being mixture quantities. The $k-\varepsilon$ model is represented in the following equations (see [11,12])

$$
\left\{\begin{array}{l}
\frac{\partial\left(\rho_{m} k\right)}{\partial t}+\frac{\partial\left(\rho_{m} k u_{j}\right)}{\partial x_{j}}=\frac{\partial}{\partial x_{j}}\left(\frac{\mu_{m t}}{\sigma_{k}} \frac{\partial k}{\partial x_{j}}\right)+p-\rho_{m} \varepsilon \\
\frac{\partial\left(\rho_{m} \varepsilon\right)}{\partial t}+\frac{\partial\left(\rho_{m} \varepsilon u_{j}\right)}{\partial x_{j}}=\frac{\partial}{\partial x_{j}}\left(\frac{\mu_{m t}}{\sigma_{\varepsilon}} \frac{\partial \varepsilon}{\partial x_{j}}\right)+\left(C_{1 \varepsilon} p-C_{2 \varepsilon} \rho_{m} \varepsilon\right) \frac{\varepsilon}{k}
\end{array}\right.
$$

$C_{1 \varepsilon}, C_{2 \varepsilon}, C_{\mu}, \sigma_{k}, \sigma_{\varepsilon}$ are the constant parameters (see [11]).

The vapor volume fraction equation is (see $[13,14])$

$$
\frac{\partial \alpha}{\partial t}+\nabla \alpha U_{m}=\frac{\dot{m}}{\rho_{l}}
$$

$\dot{m}$ is written by the following equation (see $[13,14])$

$$
\dot{m}= \begin{cases}-F_{e} \frac{3 r_{n u c}(1-\alpha) \rho_{v}}{R} \sqrt{\frac{2}{3} \frac{p_{v}-p}{\rho_{l}}} & \text { if } p<p_{v} \\ F_{c} \frac{3 \alpha \rho_{v}}{R} \sqrt{\frac{2}{3} \frac{p_{v}-p}{\rho_{l}}} & \text { if } p>p_{v}\end{cases}
$$

where $R$ is the bubble radius satisfying the Rayleigh-Plesset equation (see [5])

$$
\begin{aligned}
& R \frac{d^{2} R}{d t^{2}}+\frac{3}{2}\left(\frac{d R}{d t}\right)^{2}=\left(\frac{p_{v}(t)-p_{\infty}}{\rho_{m}}\right)+\frac{p_{g 0}}{\rho_{m}}\left(\frac{R_{0}}{R}\right)^{3 \tilde{k}}-4 \frac{v}{R} \frac{d R}{d t}-\frac{2 S}{\rho_{m} R^{\prime}} \\
& \dot{R}(0)=0 \\
& R(0)=R_{0} .
\end{aligned}
$$


Here: $p_{\infty}=p_{g 0}\left(\frac{R_{0}}{R}\right)^{3}+p_{v}-\frac{2 S}{R}$;

$p_{g 0}$ is the initial partial pressure of gas inside the bubble; $p_{v}$ is vapor pressure;

$S=0.072 \mathrm{~N} / \mathrm{m}$ water surface tension (see [5]);

$R_{0}=2.10^{-6}$;

$r_{n u c}$ is the nucleation site volume fraction, $r_{n u c}=5.10^{-4}$;

$F_{e}=50, F_{c}=0.01$ are empirical coefficients and $\tilde{k}$ is the thermal constant.

To easily solve $R$ its non-dimension $\bar{R}$ is introduced with the following relevant scales: length scale $a=R_{0}$, time scale: $\tau=\frac{R_{0}}{U_{F}}$, pressure scale: $P^{*}=\frac{1}{2} \rho U_{F}^{2}$. Then $\bar{R}$ is the solution of the following equation (see [5])

$$
\left\{\begin{array}{l}
\frac{\bar{R}}{U_{F}}=\sqrt{\frac{1}{3}\left(-C_{p}-\sigma_{v}\right)} \\
\bar{R}(0)=1
\end{array}\right.
$$

After solving $\bar{R}$ the value of $R$ is getting back by the formula:

$$
R=R_{0} \bar{R} \text {. }
$$

Here

$$
\sigma_{v}=\frac{p_{\infty}-p_{v}}{0.5 \rho_{m} U_{F}^{2}}, C_{p}=\frac{p_{S}-p_{\infty}}{0.5 \rho_{m} U_{F}^{2}}
$$

where $p_{S}$ is the local pressure on the body; The flow condition on the solid body's boundary

$$
U_{m} \cdot \vec{n}=-\vec{V} \cdot \vec{n},
$$

with $\vec{V}=\left(U_{F}, 0, W_{F}\right)$ and initial flow condition

$$
U_{m}(0)=\left(u_{1}(0), u_{2}(0), u_{3}(0)\right) .
$$

The water flow model is described by the equations (6)-(15). To solve these equations the mathematical sub-model CFD is used.

\section{COUPLE MODEL OF TWO SUB-MODELS BY UDF SUBROUTINE}

The schema of coupling model of slender body motion and water flow is shown in Fig. 4 (see [15]). The OUT-COMPUTER Programming (OPC), which calculates body's state $X=(U, W, Q, h, \vartheta)^{T}$, is executed at certain steps in CFD model. Getting the input values from OPC, CFD model is executed to find out flow velocity, pressure, density and cavity number. Using these results as well as the input data OPC calculates new body's state at the next step. Data handling between OPC and CF model is performed by means of the Memory Management System (MMS) of CFD model. The OPC's execution is fully controlled by CFD model. All variable fields are stored in MMS and read out as an initialization for OPC in the next step. The coupling model of slender body motion and water flow is performed using the following user routines:

- Junction box routine named by Outcomputer.F, which calculates body state $X=$ $(U, W, Q, h, \vartheta)^{T}$ of Eq. (5) and $\left(U_{F}, W_{F}\right)$ of Eq. (1), is executed at certain steps in CFD model. 
- User CEL Function named by Readmms.F giving back data of $U_{m}, p, \rho_{m}$ and $\sigma$ to CFD model's reading, is executed if data is requested.

- Junction Box Routine named by Createinput.F writes the values of $U_{m}, p, \rho_{m}$ and $\sigma$ to MMS.

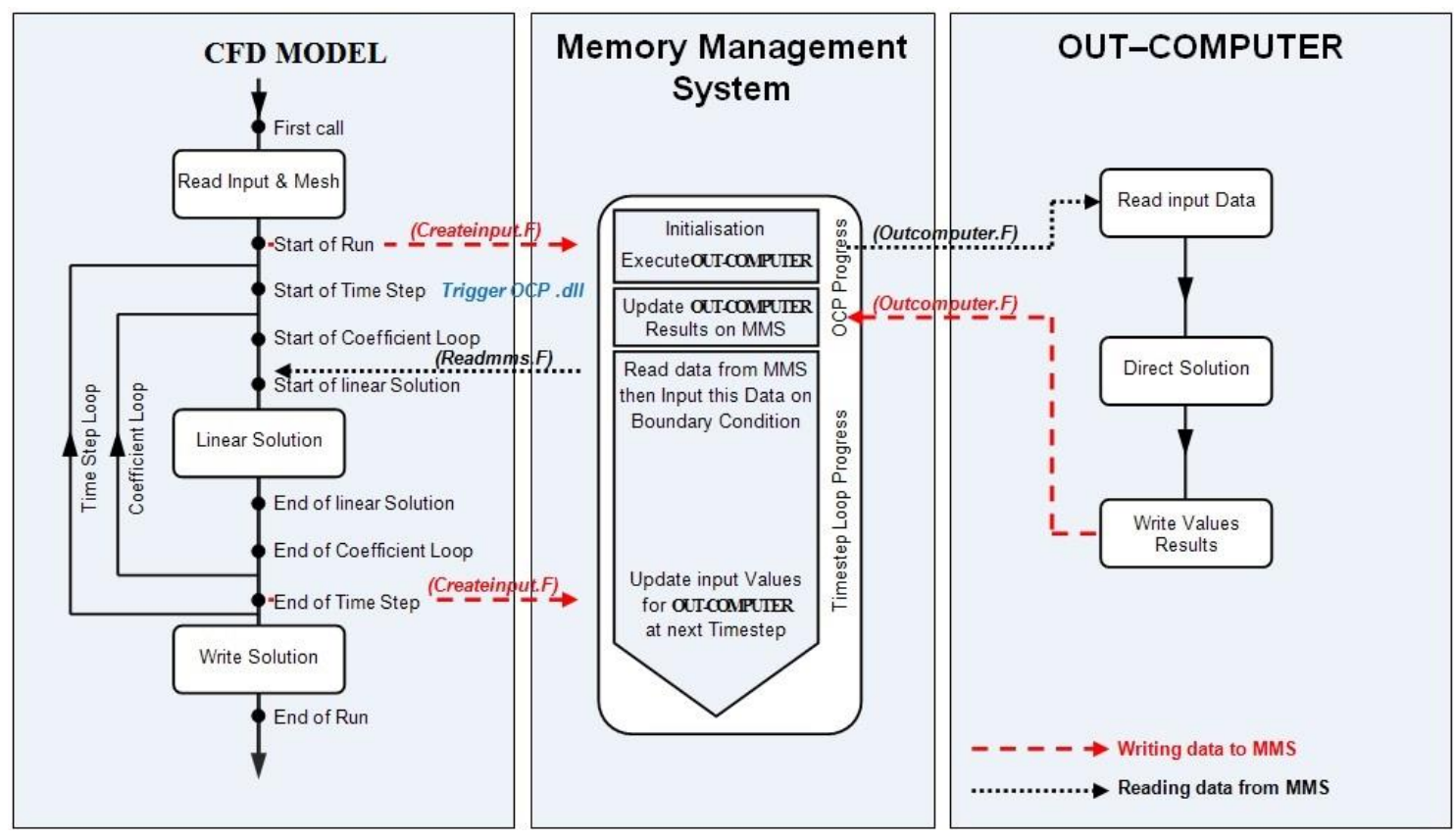

Fig. 4. Data management for a coupled OUT-COMPUTER and CFD model

Simulation process by CFD model is executed based on the diagram presented in Fig. 4. The locations, where User CEL Functions or User Junction Box Routine are called, are listed by this simulation diagram.

\section{ALGORITHM TO SOLVE THE COUPLE MODEL PROBLEM}

The coupling process of two sub-models can be described by the following steps:

1) At Start of Run: The subroutine Createinput.F is called first time, which initializes data of body's state $X_{0}=\left(U_{0}, W_{0}, Q_{0}, h_{0}, \vartheta_{0}\right)^{T}$, ambient flow pressure $p=\rho g h+p_{\text {atm }}$, where $p_{a t m}$ is the atmospheric pressure. The input parameters such as length, diameter... are saved to the MMS by this subroutine. This information is the data input of the OPC programming.

2) At Start of Time Step: OPC is called once. The input values are provided by previous time step or from subroutine Createinput.F if it is the first time step. In this step the average of ambient body's flow pressure $p$ is getting to calculate $\sigma$ (see formula (4)).

3) Then OPC is running to find out the body's state vector $X=(U, W, Q, h, \vartheta)^{T}$ of Eq. (5) and then $\left(U_{F}, W_{F}\right)$ of Eq. (1). This process is shown by the black dashed line in the right side of Fig. 4. 
4) After simulating, OPC writes back the getting state results $X=(U, W, Q, h, \vartheta)^{T}$ and $\left(U_{F}, W_{F}\right)$ as the body's data to MMS. This process is shown by the red dashed line in the right side of Fig. 4.

5) While the CFD model "coefficient loops" are performed, the subroutine Readmms.F is called some times (the number of call is depending on the Number of Time steps per Run to get body's data and $\left(U_{F}, W_{F}, Q, h, \vartheta\right)$ from MMS. This data is the input for CFD model.

6) CFD sub-program solves the equations (6)-(15) to find out the flow values $U_{m}, p$, $\rho_{m}$.

7) The results $\left(U_{m}, p, \rho_{m}\right)$ are written to MMS by the subroutine Createinput.F. These results also are the data input for OPC at next step. This process is shown by the third red line in the left side of Fig. 4.

8) Come back to step 2.

\section{SIMULATION EXPERIMENTS}

Let the body with $m=0.025091 \mathrm{~kg}, L_{1}=2.5 \mathrm{~cm}, L_{2}=11.5 \mathrm{~cm}, d=0.57 \mathrm{~cm}$, $I_{y}=1.81 \mathrm{E}^{-4} \mathrm{kgm}^{2}, d_{c}=0.18 \mathrm{~cm}, x_{c m}=6.01 \mathrm{~cm}$ the initial conditions: $U_{0}=271.2 \mathrm{~m} / \mathrm{s}$, $W_{0}=0, Q_{0}=1 \mathrm{rad} / \mathrm{s}, h_{0}=1 \mathrm{~m}, \vartheta_{0}=0$.

By the algorithm presented in the section 4 the equation system (5), (6)-(15) is solved in the time period $T=0.25 \mathrm{~s}$. In every time step, the interaction between body's motion and flow motion is expressed by the exchange of body velocities, ambient flow pressures $p$ getting by the CFD sub-program and the formula (4) calculating $\sigma$. The results are shown in Fig. 5.

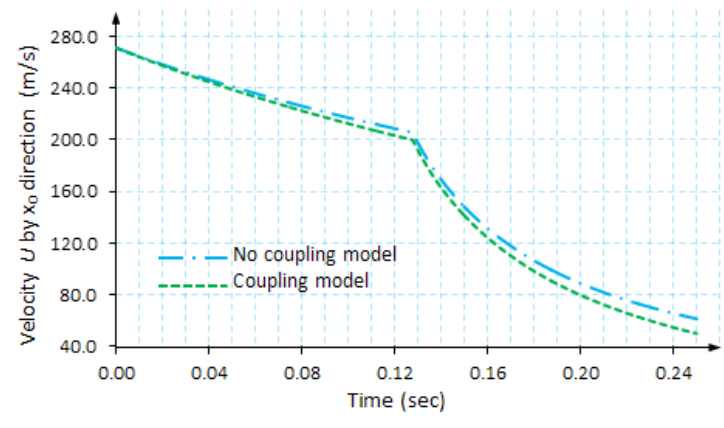

(a)

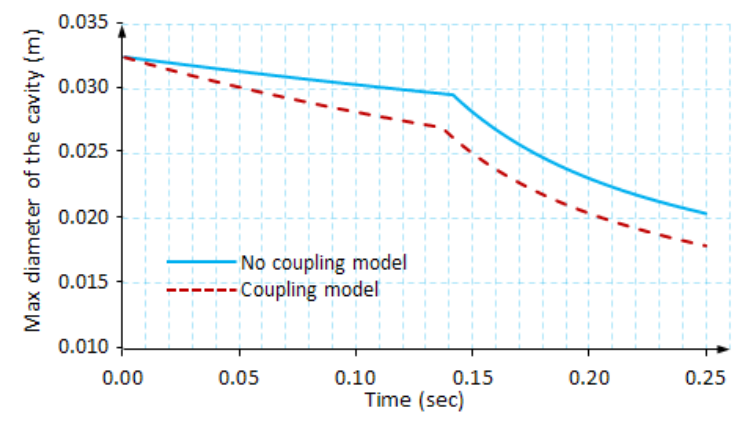

(b)

Fig. 5. Body velocity $U$ by $x_{0}$ direction (a); Time evolution of max diameter of the cavity (b)

We have done a real experimental of body running underwater with the above data (see [16]). In the real measurements we have 96 measured points of velocities $U$ by $x_{0}$ direction within the time period $T=1.6 \mathrm{~ms}$. We will consider the following comparisons.

The velocities $U$ by $x_{0}$ direction of two simulation and experiment data are presented in Fig. 6. The dot-and-dash line describes the results of the no coupling model, the dashand-dash line describes the results of the coupling model, and the solid line describes the 
experiment datas; Therefor, the results of the coupling model are exacter than the ones of no coupling model.

The pressure field around on slender body for CFD simulation is presented in Fig. 7, and the full cavity arising in very fast motion of slender body under water is shown in Fig. 8.

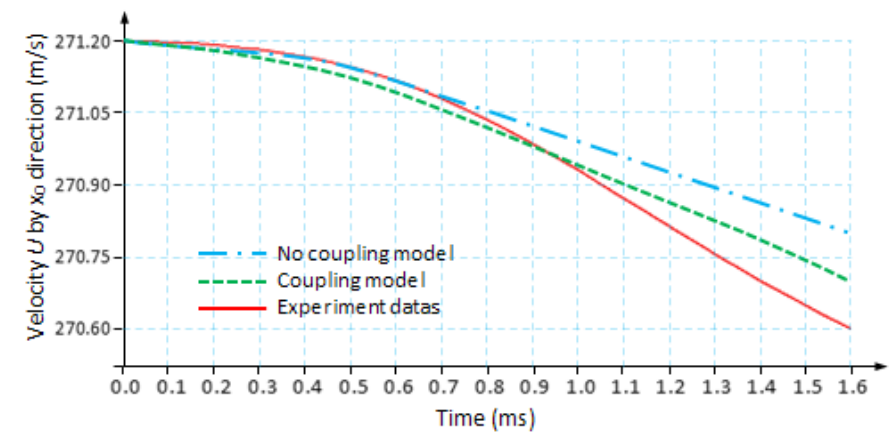

Fig. 6. Body velocity $U$ by $x_{0}$ direction

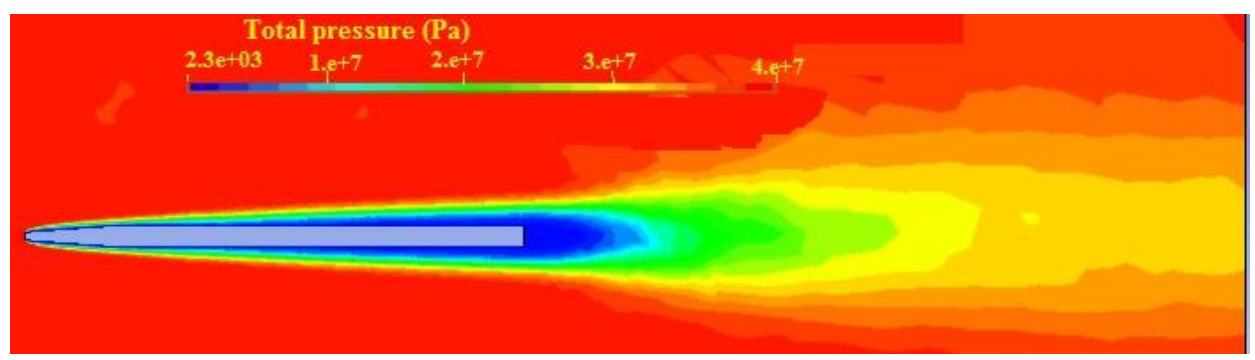

Fig. 7. The pressure field around on slender body for CFD simulation

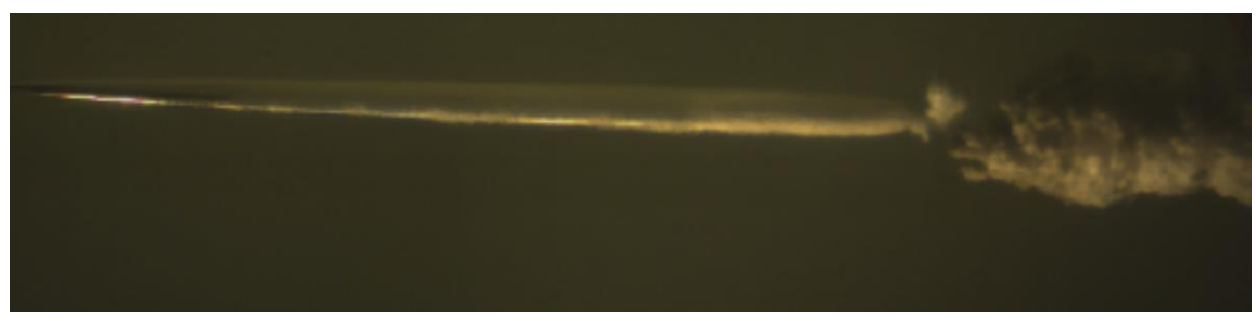

Fig. 8. The full cavity arising in very fast motion of slender body under water 


\section{CONCLUSION}

1) In the coupled model the exchanging interaction between body's fast motion and fluid flow is considered. In every time step, this interaction is expressed as follows:

- In one side, the formulas (11), (13)-(14) show that the flow velocities and pressure field are influenced by the body's velocity.

- In the other side, the formula (4) shows that the body motion is influenced by the ambient flow pressure.

2) By Fig. 6 we can see that under the interaction between body's motion and water flow the body's velocity simulated by the coupled model is lower than the other one simulated only by the sub-model 2.1 running with the ambient pressure of the flow $p=$ $\rho g h+p_{a t m}$. This causes problems for the maximum diameter of cavity decrease faster than other case, Fig. 5(b).

3) Although simulation time of coupling model is long, this model is a good tool to describe the body's fast motion underwater with considering interaction between body's motion and flow.

\section{ACKNOWLEDGEMENT}

The authors acknowledge the support by the Nafosted 107.03-2015.31 project.

\section{REFERENCES}

[1] S. S. Kulkarni and R. Pratap. Studies on the dynamics of a supercavitating projectile. Applied Mathematical Modelling, 24, (2), (2000), pp. 113-129. doi:10.1016/s0307-904x(99)00028-1.

[2] B. Milwitzky. Generalized theory for seaplane impact. Technical report, NACA-TR-1103, (1952).

[3] N. A. Son, T. T. Ha, and D. N. Hai. A super cavity model of slender body moving fast in water. In Proceedings of National Conference of Mechanics, (2014), pp. 415-420.

[4] R. Rand, R. Pratap, D. Ramani, J. Cipolla, and I. Kirschner. Impact dynamics of a supercavitating underwater projectile. In Proceedings of ASME Design Engineering Technical Conferences (DETC97), Sacramento, California, (1997). pp. 14-17, http://audiophile.tam.cornell.edu/randpdf/ahsum.pdf.

[5] J.-P. Franc and J.-M. Michel. Fundamentals of cavitation. Kluwer Academic Publisher, (2004).

[6] A. May. Water entry and the cavity-running behavior of missiles. Technical report, Navy sea Hydro ballistics Advisory Committee Silver Spring Md, (1975).

[7] I. Senocak and W. Shyy. Evaluations of cavitation models for Navier-Stokes computations. In Proceedings of the 2002 ASME Fluids Engineering Division Summer Meeting. American Society of Mechanical Engineers, (2002), pp. 395-401. doi:10.1115/fedsm2002-31011.

[8] A. K. Singhal. Multi-dimensional simulation of cavitating flows using a PDF model of phase change. In Proceedings ASME FED Meeting, Vancouver, Canada, (1997). pp. 82-97.

[9] G. Wang, I. Senocak, W. Shyy, T. Ikohagi, and S. Cao. Dynamics of attached turbulent cavitating flows. Progress in Aerospace Sciences, 37, (6), (2001), pp. 551-581.

[10] H.-L. Liu, J. Wang, Y. Wang, H. Zhang, and H. Huang. Influence of the empirical coefficients of cavitation model on predicting cavitating flow in the centrifugal pump. International Journal of Naval Architecture and Ocean Engineering, 6, (1), (2014), pp. 119-131. doi:10.2478/ijnaoe2013-0167. 
[11] W. P. Jones and B. E. Launder. The prediction of laminarization with a two-equation model of turbulence. International Journal of Heat and Mass Transfer, 15, (2), (1972), pp. 301-314. doi:10.1016/0017-9310(72)90076-2.

[12] J. W. Lindau, R. F. Kunz, D. A. Boger, D. R. Stinebring, and H. J. Gibeling. High Reynolds number, unsteady, multiphase CFD modeling of cavitating flows. Journal of Fluids Engineering, 124, (3), (2002), pp. 607-616. doi:10.1115/1.1487360.

[13] M. Morgut and E. Nobile. Numerical predictions of cavitating flow around model scale propellers by CFD and advanced model calibration. International Journal of Rotating Machinery, 2012, (2012). doi:10.1155/2012/618180.

[14] P. J. Zwart, A. G. Gerber, and T. Belamri. A two-phase flow model for predicting cavitation dynamics. In Fifth International Conference on Multiphase Flow, Yokohama, Japan, (2004). pp. 74-83.

[15] E.-A. Reinecke, S. Kelm, W. Jahn, C. Jäkel, and H.-J. Allelein. Simulation of the efficiency of hydrogen recombiners as safety devices. International Journal of Hydrogen Energy, 38, (19), (2013), pp. 8117-8124. doi:10.1016/j.ijhydene.2012.09.093.

[16] D. N. Hai, N. T. Thang, and T. T. Phuong. Experimental measurement of supercavity around body moving into water. In Proceedings of the 4th National Conference on Measurement Science and Techniques, Hanoi, Vietnam, (2015). pp. 740-747. (in Vietnamese). 